\title{
URETERORENOSCOPY, A REVIEW OF FIRST THREE YEARS OF EXPERIENCE FOR URETERIC STONE DISEASE.
}

1. FCPS

Department of Urology

Sargodha Medical College Sargodha.

2. FCPS

Head of Department of Urology Sargodha Medical College Sargodha.

3. M.Phil. Biochemistry Department of Biochemistry Ayub Medical College Abbottabad.

Correspondence Address:

Dr. Saif Ud Din Awan

Department of Urology

Sargodha Medical College

Sargodha.

saifuddinawan@yahoo.com

Article received on:

06/09/2016

Accepted for publication:

15/06/2018

Received after proof reading:

30/09/2019

\begin{abstract}
Saif Ud Din Awan', Ahmad Nawaz Bhatti ${ }^{2}$, Ayesha Naureen Awan ${ }^{3}$
ABSTRACT... Objectives: Ureterorenoscopy is a diagnostic and therapeutic modality, used for different ureteral and renal pathologies. It has virtually replaced open surgery for ureteric calculi. Study Design: Observational. Setting: Urology Department of Sargodha Medical College, Sargodha. Period: 2011-2015. Material and Methods: All cases who underwent URS for stone disease during the study period were included in the study. The initial criterion was a bit strict, due to minimum experience, which limited only clinically fit patients with no co-morbid illnesses, with stone size of $1 \mathrm{~cm}$ or less, located in middle or preferable in distal ureter, single stone was also preferred, but later the criterion was relaxed with gain confidence. The procedures were performed under general anesthesia using $\mathrm{R}$ Wolf Ureterorenoscope. Results: The total number of cases included in the study were 332 of which 189 were males and 143 were females. Average age being 42.82 years. The total number of stones managed were 354 (in 22 cases the stones were bilateral). The average size of stones was $1.2 \mathrm{~cm}$. DJ stent was used in 78 cases. Complications were observed in 14.76 percent cases $(2.71 \%$ major and $12.05 \%$ minor). No deaths occurred in the study group. Conclusion: Uretero renoscopy is an excellent tool for the management of ureteral stone disease so much so that these cases, after sufficient experience, can be managed as day cases. The complication rate can be controlled by careful patient selection. Being intuitive and stopping the procedure on the very first hint of something going wrong like the scope being stuck or requiring excessive force. Strictures should not be traversed without adequate prior dilatation. The operator should have sufficient experience with open surgery.
\end{abstract}

Key words: $\quad$ Complication after Ureterorenoscopy, Stone-Free Rates Ureterorenoscopy, Urolithiasis.

Article Citation: Awan S, Bhatti AN, Awan AN. Ureterorenoscopy, a review of first three years of experience for ureteric stone disease. Professional Med J 2019; 26(10):1712-1718. DOI: 10.29309/TPMJ/2019.26.10.4133

\section{INTRODUCTION}

Ureteroscopy is a diagnostic and therapeutic modality, used for different ureteral and renal pathologies. Ureteroscopy was first carried out in 1929 by Young and Mckay using a cystoscope in grossly dilated ureter $^{1,2}$, however rigid ureteroscope was introdused in clinical practice in late 1970 s. $^{3}$

Since its clinical introduction in 1982 by PerezCastro Ellendt and Martinez-Pineiro, ureteroscopy has experienced an impressive development due to the technical improvements of new and smaller urological armamentarium..$^{3,4}$ In the last decade ureteroscopy has become an outstanding breakthrough in the diagnosis and treatment of different ureteral and renal pathologies.

Ureteral perforation or avulsion, bleeding, and urinary tract infection are amongst the important complications of the procedure. ${ }^{4}$ and may require nephrectomy in a few cases.

Since its introduction, Ureteroscopy has become a widely practised method for the diagnosis and treatment of pathologies of both the ureter and the intrarenal collecting systems. ${ }^{5}$ Its use in the developing countries like Pakistan is on an increase and smaller cities are also turning to the latest treatment modalities in patient care.

\section{MATERIALS AND METHODS}

\section{Inclusion criterion}

Initially ureteric calculus of upto $1 \mathrm{~cm}$ located in the lower and middle ureter were managed by Ureterorenoscopy, but later, with experience, larger stones (up to $1.8 \mathrm{~cm}$ ) and those located in the proximal ureter were also subjected to this modality successfully. cases of bilateral / solitary renal obstructive uropathy were also managed by this modality in order to by pass the obstruction 
by removal of the stone and/or placement of DJ stent were carried out once sufficient expertise were attained.

All the cases of ureteric calculus which were managed by this modality are included in this study so as to determine the out come and improvement in the skills over the period under study.

\section{Exclusion Criterion}

Cases with dilated collecting system and no visible cause of obstruction having raised serum urea and creatinine levels were also managed by this modality for diagnostic purpose and an attempt to pass a DJ stent so as to relieve the pressure from the collecting system. URS carried out for diagnostic purposes are not included in this study.

\section{Equipment}

Semi rigid ureterorenoscope ( $\mathrm{R}$ Wolf, $12^{\circ}$ )

Karl storz Xenon 300 light source.

Karl Storz tricam SL NTSC camera and monitor Guide wire (Tefloan coated, 0.035”, $150 \mathrm{~cm}$ ).

Double J 'pigtail stents (6Fr, $26 \mathrm{~cm})$

Pneumatic lithotripter (Pulselith).

\section{METHOD}

All the procedures were carried out under general anesthesia with muscle relaxation.

The urinary bladder was drained by $6 \mathrm{fr}$ peadiatric feeding tube.
$\mathrm{R}$ Wolf Ureterorenoscope was used along with a karl Storz light source, positive pressure irrigation source with normal saline was used. The URS was passed into the urinary bladder the anatomy was identified and orifices of the ureter were located. Guide wire was passed into the ureter and the tip of URS was guided over it to enter the ureter (later with experience we are passing the URS in to the ureter without the assistance of guide wire). The instrument was gradually advanced up to the stone and pneumatic lithotripsy was used for stone fragmentation and it was made certain that all the fragments were about $2 \mathrm{~mm}$ in size or smaller, for spontaneous expulsion. DJ stent was used if the stone fragments were considered to be large in case of hard stones or for heavy stone burden due to larger stone size. The stent, if placed, was removed after radiological confirmation of passage of all stone fragments around 10th post-operative day (range 6 to 28 days).

Variables of interest were collected for the patients under study from the hospital record and analyzed using SPSS 10.

\section{RESULTS}

The total number of cases included in the study were 332 of which 189 were male and 143 were female.

The average age of the patients who under went URS from 2011 to 2014 was 42.82 (range 17 years to 75 years) the breakdown is given in the Table-l.

\begin{tabular}{|c|c|c|c|c|c|}
\hline \multicolumn{2}{|c|}{ Years } & 2011-2012 & 2012-2013 & 2013-2014 & 2011-2014 \\
\hline \multicolumn{2}{|l|}{ Cases (n) } & 93 & 111 & 128 & 332 \\
\hline \multicolumn{2}{|l|}{ Age (years) } & 41.29 & 43.68 & 43.61 & 42.82 \\
\hline \multirow{2}{*}{ Gender } & Male & 56 & 62 & 71 & 189 \\
\hline & Female & 37 & 49 & 57 & 143 \\
\hline \multicolumn{2}{|l|}{ Stone size $(\mathrm{cm})$} & 0.98 & 1.2 & 1.3 & 1.2 \\
\hline \multirow{3}{*}{ Stone location } & Upper & 2 & 8 & 17 & 27 \\
\hline & Middle & 13 & 20 & 34 & 67 \\
\hline & Lower & 84 & 92 & 84 & 280 \\
\hline \multirow{2}{*}{ Laterality } & Right & 51 & 61 & 61 & 173 \\
\hline & Left & 48 & 59 & 74 & 181 \\
\hline \multicolumn{2}{|c|}{ Procedure time (mins) } & 39.43 & 31.51 & 18.65 & 29.64 \\
\hline \multirow{2}{*}{\multicolumn{2}{|c|}{$\begin{array}{l}\text { DJ stent used } \\
\text { Stone free Rate (percent) }\end{array}$}} & 27 & 25 & 26 & 78 \\
\hline & & 98.92 & 99.10 & 98.44 & 98.80 \\
\hline \multirow{2}{*}{ Complications } & Major & 3 & 4 & 2 & 9 \\
\hline & Minor & 14 & 13 & 13 & 40 \\
\hline
\end{tabular}


A total of 354 stones were operated of which 22 were bilateral.

The location of stones in majority of the cases was the lower third of the ureter $(n=280)$ Average size $1.2 \mathrm{~cm}$ (range 0.5 to $1.8 \mathrm{~cm}$ ).

DJ stent was used in 78 cases and were removed on an average of 10 days (range 6 to 28 days)

Average time taken by the procedure was 29.64 minutes which during the first year was 39.43 mins and improved to 18.65 mins during the last year under study.

Stone free rate was 98.80 percent

Complication rate of 14.76 percent was observed (Table-II) of which 2.71 were major (including perforation, ureteric avulsion and stent migration) while 12.05 were minor (including loin discomfort, fever, hematuria, transient rise in renal function, and proximal stone migration).

However no deaths occurred amongst the study group.

\begin{tabular}{|c|l|c|c|}
\hline Grade & \multicolumn{1}{|c|}{ Complication } & No. & Percentage \\
\hline I & Mucosal injury & 13 & 3.92 \\
\hline I & Hematuria & 8 & 2.41 \\
\hline I & Fever & 9 & 2.71 \\
\hline I & Post obstructive diuresis & 2 & 0.60 \\
\hline I & $\begin{array}{l}\text { Transient elevation in } \\
\text { renal functions }\end{array}$ & 1 & 0.30 \\
\hline I & Urine retention & 0 & 0.00 \\
\hline I & Mechanical failure & 0 & 0.00 \\
\hline II & Urinary tract infection & 7 & 2.11 \\
\hline IIIa & Proximal stone migration & 4 & 1.20 \\
\hline IIIb & Perforation & 2 & 0.06 \\
\hline IIIb & $\begin{array}{l}\text { Extravasation and open } \\
\text { conversion }\end{array}$ & 1 & 0.30 \\
\hline IIIb & Stent migration & 1 & 0.30 \\
\hline IVa & $\begin{array}{l}\text { Myocardial infarction, } \\
\text { Pulmonary embolism }\end{array}$ & 1 & 0.30 \\
\hline IVb & Urosepsis & 0 & 0.00 \\
\hline V & $\begin{array}{l}\text { Death } \\
\text { Table-II. The complications and their } \\
\text { according to the MCCS }\end{array}$ \\
\hline MCcs: Modified Clavien classification system \\
\hline
\end{tabular}

Professional Med J 2019;26(10):1712-1718.

\section{DISCUSSION}

Ureteroscopy is a diagnostic and therapeutic modality, used for different ureteral and renal pathologies. Young and Mckay were the pioneers of Ureteroscopy having used it in a grossly dilated ureter in 1929 by using a cystoscope ${ }^{1,2}$, how ever its routine clinical use started in the late 1970 s with a rigid ureteroscope. ${ }^{3}$ once in clinical use the shortcommings of ureteroscope were recognised and gradual refinements were made in its design. Light source was improved so as to make it more bright and reduce its heating effect on the tissues. making the procedure easy for the surgeon and safe for the patient. Its wide clinical use of ureteroscopy started in 1982 by PerezCastro Ellendt and Martinez-Pineiro. Thereafter it has experienced an impressive development due to the advancements in the fields of optics and electronic. Now newer and smaller urological instruments are being designed and produces. ${ }^{3,4}$ In the last decade ureteroscopy has become the procedure of choice in the diagnosis and treatment of different ureteral and renal diseases.

With the introduction of fibre-optics the endoscopes have started shrinking in size. we can now introduce a $2-3 \mathrm{~mm}$ semi-rigid ureteroscope up to the renal pelvis via the urethra with excellent views and an instrument channel large enough for both energy sources to fragment calculi and for retrieval devices to harvest the fragmented stones. The development of the flexible ureterorenoscope and intracorporeal laser technology has allowed small intrarenal stones to be treated from below, this technique has threatened the role of percutaneous renal surgery for such calculi. Some authors consider that developments in ureteroscope design and technique of ureterorenoscopy have determined the success of diagnostic and therapeutic use and made it much safer. . $^{5,7}$

The use of Ureteroscopy in conjunction with laser lithotripsy is very successful for nearly all ureteric calcui and, even though ESWL is considered the first choice modality for most renal and ureteric stones, its relatively inferior success rates and associated complications exclude it as choice for every single patient. Moreover, lack of 
availability of ESWL facilities encourage the use of endoscopic stone treatments. Ureteroscopy is considered to be first line therapy in the obese patient, those on anticoagulation therapies and in pregnant ladies. Lithotripsy method during ureteroscopy may have effect on complication rate. Georgescu et.al, in a large study showed that electrohydraulic fragmentation had $3.9 \%$ complication rate, however this rate reduced to $3 \%$ and $2.8 \%$ with pneumatic or laser lithotripsy correspondingly. although it was not statistically significant. $^{8}$

General anesthesia is used for Ureteroscopy and it gradually being shifted to the domain of day case procedures. Although all patients undergoing ureteroscopy would receive a ureteric stent post-operatively but the due to more experience and available studies on the subject the routine use stent is discouraged and mre and more ureteroscopy and ureterorenoscopy can be performed "stentless".

sometimes it is difficult to introduce ureterorenoscope into the ureter, in such cases a stent can be inserted to allow passive ureteric dilatation over two weeks allowing easier ureteroscopic stone fragmentation and removal.

Our study shows that the average age of patients undergoing URS in our centre was 42. years (range 17 to 75 years) which is slightly on the lower side compared to other international studies. The probable reason may be that Pakistan is located in the stone forming belt and due to high temperature and low consumption of water stones are seen in relative younger age group. Moreover our intentional case selection during the initial period excluded the older age group because of the co-morbid diseases which however with gain of experience and confidence changed and age and co-morbidities were no more an exclusion criterion.

There were 189 male and 143 females in our study (Figure-1), with a male to female ratio of about $2: 1$. The reason for this being that the male perform hard jobs in the open sun and are therefore relatively more prone to dehydration and concentration of urine thus forming gravel and stones. Moreover our society is male centered and poor, therefore females are not usually brought to the hospitals for treatment rather they are taken to the quacks.

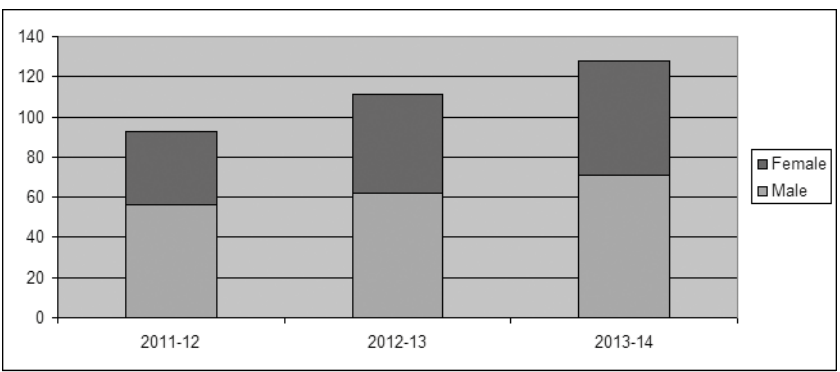

Figure-1. Increase in number of cases managed by Ureterorenoscopy

A total of 354 stones were managed during the period of study in 332 cases, 22 patients having bilateral stones. the average size of stones managed was $1.2 \mathrm{~cm}$ (range 0.5 to $1,8 \mathrm{~cm}$ ) the trend which could be identified from the table 1 is that we selected small sized, distal stones during the early part of our study where as later larger and more proximal stones were also managed by URS.

Double ' $J$ ' stent also referred to as double pigtail stent was used in cases where, large stone burden or large fragments of stones (specially hard stones) were suspected to produce obstruction. 'Stein strassae' was observed in two cases but due to antisepatory placement of DJ stent the patients recovery went smooth with a little assistance with alpha blockers and analgesics. the stent was removed once the X-Ray showed complete passage of stones (up to 4 weeks). A case of proximal stent migration was also seen in which the stent was removed using URS and grasper.

Average time taken by the procedure was 29.64 minutes (Figure-2) which during the first year was 39.43 mins and improved to 18.65 mins during the last year under study. This was achieved due to experience gained in handeling of the instrument, recognition of anatomy and cannulation of the ureter by the scope. 


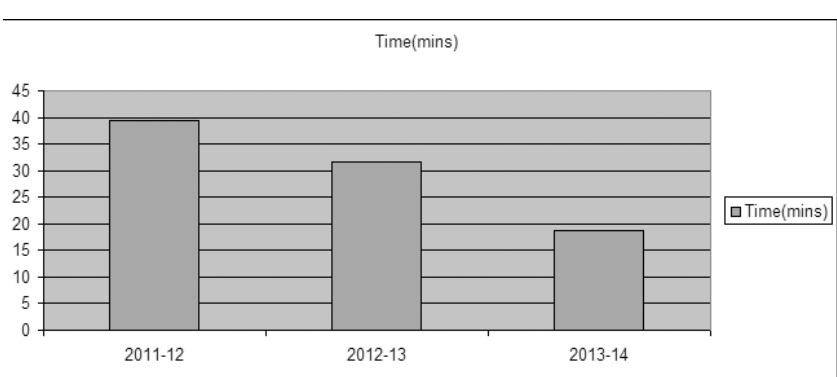

Figure-2. Reduction in average Time per cases

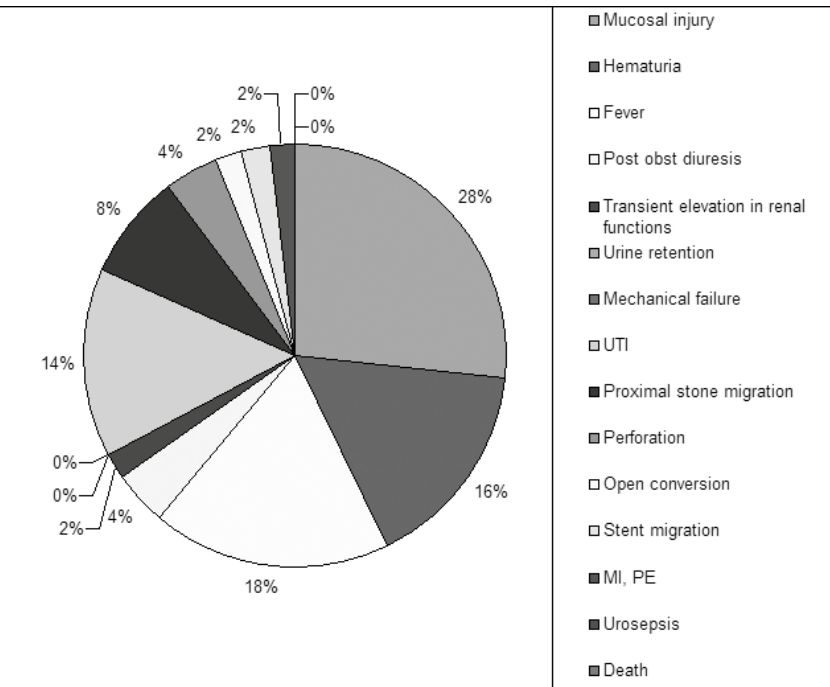

Figure-3. Pie chart showing complications encountered in the study.

In our study a stone free rate of 98.24 percent was observed approaching nearly 100 percent during the first year due to meticulous case selection.

Ureteroscopy has its own set of complications divided into minor and major; minor complications include asymptomatic ureteral perforations, ileus, and fever, whereas major complications, include tears, perforations during basketing, and, rarely, avulsions of the ureter (often associated with stone extractions), intussusception, and sepsis. Necrosis of ureteral segments after ureteroscopy for stone removal has also been reported..$^{9-12}$

An overall complication rate of 14.76 percent was observed in our study (Table-I, Figure-1) of which 2.71 were major (including perforation, ureteric avulsion and stent migration) while 12.05 were minor (including loin discomfort, fever, haematuria, transient rise in renal function, and proximal stone migration) Table-
II. However, Geavlete and colleagues reported the intraoperative complication rate was $5.6 \%$ for proximal calculi and $3.6 \%$ for distal stones ${ }^{5}$, which is much less than our result, but taking into consideration that it was our initial experience / learning phase the results can be expected to improve to international standards over time.

The commonest problem encountered after ureteroscopy is post-operative loin discomfort, which may last for a few days and is treated with simple analgesia. In our study the reason found to be the cause of loin pain, was longer duration of the procedure with consequently more use of irrigation fluid under pressure causing renal/ perirenal extravassation/edema, more over it was also observed in the patients in whom DJ stent were used. The reason in these cases was back flow of urine through the stent during mituration and irritation of the trigon by the coiled tip of the stent. However, fever and haematuria are relatively more common but are clinically not significant with no long term consequences.

Cases of Urinary tract infections were significantly less common than anticipated due to regular use of prophylactic single shot antibiotic and if UTI did occur was easily treated with standard antibiotic therapy. More over a preoperative sample was collected for culture and in cases of urosepsis culture specific antibiotic therapy was commenced with good outcome.

Conversion to open surgery is required for rare cases of ureteric avulsion. Moreover the discrepancy between ureteroscope size and calibre of the patient's ureter also contribute to ureteric avulsion. ${ }_{13}$ we encountered one such case during the first year of the study. The patient had a stricture which was being negotiated and excess force was applied which resulted in ureteric avulsion. The complication was identified per operatively and a guide wire was place into the proximal ureter. The procedure was converted to open. The distal ureter was found to be non viable as it was completely de-gloved with no viable blood supply. Boari's flap and psoas hitch was performed and the distal end of the proximal ureter was anastomosed to the bladder over a 
DJ Stent, which was removed at 6 weeks. The post operative results as assessed on follow up at 6 months and one year showed satisfactory renal anatomy and function. More over ureteric perforations were recognized in two patients and DJ stent was placed without the requirement of opening, the follow up results were satisfactory. Proper ureteroscopic handling is the key in prevention of these catastrophic complications. The reconstruction in case of ureteric avulsion with loss of long segment of the upper ureter, when end-to-end anastomosis is not technically feasible and it presents a challenge to the urological surgeon. In such patients autotransplantation of the kidney is thought to be a favored management strategy. ${ }^{14}$ Other possible techniques used for ureteral reconstruction include psoas hitch, Boari flap, or a combination of both, ileal interposition, transureteroureterostomy, permanent nephrostomy, appendix or colon interposition, renal descensus, ureterocalicostomy, and pyeloureterostomy plus greater omentum investment outside the avulsed ureter, if these modalities are not possible than as a last resort nephrectomy may have to be performed. ${ }^{4,6,14-18}$

due to the use of small caliber endoscopes the long term complication of uretreic stricture is not frequently observed and can be expected in less than $1 \%$ of the patients. ${ }^{19}$ However in our study no case of post operative/iatrogenic stricture was identified with a minimum follow up period of one year.

No deaths occurred in the study group.

Here are a few technical tips for the beginners (1) you should insert a guidewire into the ureter prior to insertion of ureteroscope; (2) if you feel it difficult to advance the ureteroscope despite full visualization of the lumen and feel that excessive force is required, they should withdrawn the URS immediately, replaced it with a smaller calibre instrument after ureteral dilation or place a ureteral stent and repeat the procedure about a couple of weeks later, to allow for ureteral dilation; (3) if you see ureteral stricture, dilate it before insertion of the scope or incision of stricture should be performed; (4) if you plan to perform proximal ureteroscopy lubricant should be applied along the entire length of the shaft of the scope; and (5) if you feel that scope is impacted or you need more power for extraction of scope, a second semirigid ureteroscope should be passed into the bladder beside the first and the situation is evaluated. ${ }^{9,14}$

\section{CONCLUSION}

Uretero renoscopy is an excellent modality for the management of lower and middle ureteric calculi, which has virtually replaced open surgery. Although the learning curve to master this procedure is long but still it out weighs the benefits. With experience the operative time and complication rate is reduced and conversion to open surgery is almost negligible.

\section{Copyright $\subseteq 15$ June, 2018.}

\section{REFERENCES}

1. Young HH, McKay RW. Congenital valve obstruction of the prostatic urethra. RR Donnelley; 1929.

2. Dublin N., Razack, AH, "Avulsed ureter," ANZ Journal of Surgery, 2003:Vol. 73, no. 4: pp. 254-255.

3. Pérez-Castro EE, Martinez-Pineiro JA. Ureteral and renal endoscopy. A new-approach. European urology. 1982;8(2):117-20.

4. Alapont JM, Broseta E, Oliver F, Pontones JL, Boronat F, Jiménez-Cruz JF. Ureteral avulsion as a complication of ureteroscopy. International braz j urol. 2003 Feb;29(1):18-23.

5. Geavlete P, Georgescu D, Niț̆ G, Mirciulescu V, Cauni V. Complications of 2735 retrograde semirigid ureteroscopy procedures: a singlecenter experience. Journal of endourology. $2006 \mathrm{Mar}$ 1;20(3):179-85.

6. Lutter I, Molcan T, Pechan J, Daniel I, Wagenhoffer R, Weibl P. Renal autotransplantation in irreversible ureteral injury. Bratislavske lekarske listy. 2002;103(11):437-9.

7. Elashry OM, Elgamasy AK, Sabaa MA, Abo-Elenien M, Omar MA, Eltatawy HH, El-Abd SA. Ureteroscopic management of lower ureteric calculi: a 15-year single-centre experience. BJU international. 2008 Oct;102(8):1010-7. 
8. Georgescu D, Mulțescu R, Geavlete B, Geavlete P. Intraoperative complications after 8150 semirigid ureteroscopies for ureteral lithiasis: risk analysis and management. Chirurgia (Bucur). 2014;109(3):36974.

9. Gupta V, Sadasukhi TC, Sharma KK, Yadav RG, Mathur R, Tomar V, Yadav SS, Priyadarshi S, Gupta P. Complete ureteral avulsion. The Scientific World Journal. 2005;5:125-7.

10. Taie K, Jasemi M, Khazaeli D, Fatholahi A. Prevalence and management of complications of ureteroscopy: a seven-year experience with introduction of a new maneuver to prevent ureteral avulsion. Urology journal. 2012 Feb 27;9(1):356-60.

11. Bernhard PH, REDDY PK. Retrograde ureteral intussusception: a rare complication. Journal of endourology. 1996 Aug;10(4):349-51.

12. Kijvikai K, Haleblian GE, Preminger GM, de la Rosette J. Shock wave lithotripsy or ureteroscopy for the management of proximal ureteral calculi: an old discussion revisited. The Journal of urology. 2007 Oct 1;178(4):1157-63.

13. Shrestha B, Baidya JL. An unusual experience with ureterorenoscopy: a case report. Journal of Nepal Health Research Council. 2012 Aug 8.
14. Shokeir AA. Interposition of ileum in the ureter: a clinical study with. British journal of urology. 1997 Mar;79(3):324-7.

15. Ge C, Li Q, Wang L, Jin F, Li Y, Wan J, Lan W, Liang $P$. Management of complete ureteral avulsion and literature review: a report on four cases. Journal of endourology. 2011 Feb 1;25(2):323-6.

16. Ordon M, Schuler TD, Honey RJ. Ureteral avulsion during contemporary ureteroscopic stone management:"the scabbard avulsion". Journal of endourology. 2011 Aug 1;25(8):1259-62.

17. Gao P, Zhu J, Zhou Y, Shan Y. Full-length ureteral avulsion caused by ureteroscopy: report of one case cured by pyeloureterostomy, greater omentum investment, and ureterovesical anastomosis. Urolithiasis. 2013 Apr 1;41(2):183-6.

18. Deyl RT, Averbeck MA, Almeida GL, Pioner GT, Souto CA. Appendix interposition for total left ureteral reconstruction. Journal of pediatric urology. 2009 Jun $1 ; 5(3): 237-9$.

19. Ahn ST, Kim JH, Park JY, Moon DG, Bae JH. Acute postoperative pain after ureteroscopic removal of stone: incidence and risk factors. Korean journal of urology. 2012 Jan 1;53(1):34-9.

\begin{tabular}{|c|c|c|}
\hline \multicolumn{3}{|c}{ AUTHORSHIP AND CONTRIBUTION DECLARATION } \\
\hline Sr. \# & Author-s Full Name & \multicolumn{1}{|c|}{ Contribution to the paper } \\
\hline 1 & Saif Ud Din Awan & $\begin{array}{l}\text { Planning, Performing operative } \\
\text { procedures, conducting the } \\
\text { study and write up. } \\
\text { Planning, Performing operative } \\
\text { procedures and supervision } \\
\text { fo study. } \\
\text { Literature review, Write up, } \\
\text { Proof reading, Data analysis, } \\
\text { review of paper. }\end{array}$ \\
\hline 3 & Ahesha Naureen Awan & Ahmad Nawaz Bhatti \\
\hline
\end{tabular}

